\title{
Management of a patient on dual antiplatelet therapy presented for living donor liver transplant
}

\author{
Marina Moguilevitch ${ }^{1,2^{\star}}$, Ellise Delphin ${ }^{1,2}$, James Widyn $^{1}$, David Turk $^{1}$ \\ ${ }^{1}$ Department of Anesthesiology, Montefiore Medical Center, Bronx, USA; \\ *Corresponding Author: mmoguile@montefiore.org \\ ${ }^{2}$ Albert Einstein College of Medicine, Bronx, USA
}

Received 3 March 2013; revised 11 April 2013; accepted 20 April 2013

Copyright (c) 2013 Marina Moguilevitch et al. This is an open access article distributed under the Creative Commons Attribution License, which permits unrestricted use, distribution, and reproduction in any medium, provided the original work is properly cited.

\begin{abstract}
We report a case of a patient who underwent Living Donor Liver Transplantation (LDLT) while being continued on his dual antiplatelet regimen of clopidigrel and aspirin perioperatively. The patient had two drug eluting stents for coronary artery disease placed eight months prior to surgery. Preoperative thromboelastography and platelet mapping showed normal clot formation. Based on these tests it was determined that preoperative platelet transfusion was not necessary. The surgery proceeded relatively uneventfully. The patient received 1 unit of packed red blood cells intraoperatively and continued on aspirin during the postoperative period with no evidence of bleeding or cardiac ischemia.
\end{abstract}

Keywords: Liver Transplantation; Antiplatelet Drugs; Blood Transfusion; Coronary Artery Disease

\section{INTRODUCTION}

Successful Living Donor Liver Transplantation (LDLT) was described by surgeons at the Wyler's Children's Hospital (University of Chicago) in the late 1980s. Its use benefitted a predominantly pediatric population for the next decade [1-3]. In the 1990s, LDLT was extended to adult patients with results similar to those of cadaveric liver transplantation. Continual progress in the fields of hepatology, liver transplantation and transplant anesthesiology over the past two decades have made the expansion of recipient inclusion criteria possible. Many patients previously deemed unsuitable for LDLT due to age and co-morbid disease are now eligible for the procedure.

In addition, the care of patients with high risk cardiac disease undergoing non-cardiac surgery has been studied in an evidence based, systematic manner. A Scientific Statement from AHA/ACCF (American College of Cardiology Foundation) on Cardiac Disease Evaluation and Management Among Kidney and Liver Transplant Candidates discusses in detail the preoperative work up for this patient population with high risk cardiac disease [4]. Unfortunately, there are no specific recommendations for patients with coronary stents. ACCF/AHA Practice Guidelines for percutaneous coronary intervention strongly endorse the continuation of dual antiplatelet therapy in patients with drug eluting stents [5]. It is recommended that non-emergent surgery that would require discontinuation of the therapy be postponed for at least a year due to the high incidence of coronary thrombosis that occurs with discontinuance of the drugs. The ASA has adopted these guidelines in the Practice Alert for Perioperative Management of Patients with Coronary Artery Stents [6].

Liver transplantation poses the potential for massive blood loss due to preexisting coagulopathy related to hepatic failure and complex major surgery involving multiple vascular anastomoses. Liver transplantation in a patient with dual antiplatelet therapy is considered an especially high risk procedure because of the increased chance of perioperative bleeding complications requiring massive transfusion of blood and blood products.

In our case the LDLT was warranted because patient's condition was deteriorating significantly during the recommended waiting time of at least one year post stent insertion. That is why after careful preoperative assessment of the platelet function the decision was made to proceed with the surgery.

\section{CASE PRESENTATION}

A 58 years old male with end stage liver disease caused by primary sclerosing cholangitis was to receive a LDLT. He had a history of coronary artery disease and 
had undergone cardiac catheterization and the placement of two drug eluting stents in anterior descending and circumflex arteries eight months prior to presentation for surgery. Past surgery included laparoscopic appendectomy and cholycystectomy as well as a surgical biopsy of a benign right sided diaphragmatic mass. His medications regimen consisted of clopidogel and aspirin. He did not take any medication the day of surgery. Physical examination revealed jaundice, a normal airway examination and normal cardiac and pulmonary examinations.

His laboratory studies revealed: Hgb $12.4 \mathrm{mg} / \mathrm{dl}$, total bilirubin/direct bilirubin 7.2/4.8 mg/dl respectively, BUN $7 \mathrm{mg} / \mathrm{dl}$, Creatinine $0.6 \mathrm{mg} / \mathrm{dl}$, AST $59 \mathrm{u} / \mathrm{ml}$, ALT 83 u/ml, PTT 29.8 sec, INR 1.3, Platelets 330,000. Preoperative platelet mapping revealed normal clot formation and strength and no suppression to both drugs. TEG revealed normal clot strength, fibrinogen formation, clot growth rate and stability (Figure 1). Transthoracic echocardiogram showed normal left ventricular function. A stress echo revealed a mild fixed defect involving the inferior wall and no ischemia induced wall motion abnormalities.

Preoperative platelet transfusion was deemed unnecessary due to normal platelet quantity and adequate qualitative platelet function.

Standard ASA monitors were placed. After pre-oxygenation, anesthesia was induced with midazolam $4 \mathrm{mg}$, fentanyl $100 \mathrm{mcg}$, lidocaine $60 \mathrm{mg}$, propofol $140 \mathrm{mg}$, cisatracurium $20 \mathrm{mg}$. The patient was intubated orally with an 8.0 cuffed endotracheal tube. Subsequently, two large bore intravenous lines, a right radial arterial line arterial line, a right internal jugular 8.5 French introducer with Swan Ganz catheter was placed. A transesophageal echo (TEE) was used during the case. The patient remained stable throughout the dissection phase.

Blood pressure and pulmonary artery pressure (PAP) remained at baseline, 130/80 mmHg and 22 - 26/12 - 17 $\mathrm{mmHg}$ respectively. Estimated blood loss was 350 cc with a decrease in hematocrit from $37.1 \%$ to $30 \%$. A norepinephrine infusion was started in preparation for cross clamping of the inferior vena cava at the rate of 0.1 - $0.15 \mathrm{mcg} / \mathrm{kg} / \mathrm{min}$. During the anhepatic phase, one unit of packed red blood cells was transfused when the hematocrit reached 26.5\%. During reperfusion, ST segment depressions were noted in inferior-lateral leads, with simultaneous elevation of the PAP to 50/30 mmHg. No changes were noted on TEE. A nitroglycerin infusion was started with resolution of the ST segment depressions and normalization of the PAP. The estimated blood loss for the entire procedure was 700 cc. The patient received one unit of packed red blood cells.

The patient was extubated on POD 1 and placed on aspirin after consultation with cardiologist. His postoperative course was uneventful, and he was discharged home on POD 15 in stable condition.

\section{DISCUSSION}

Coagulopathy secondary to end stage liver disease occurs frequently in patients presenting for liver transplantation. During the operation, hemorrhage may occur due to a pre-existing hypocoagulable state related to decrease in synthesis of a majority of coagulation factors and proteins involved in fibrinolysis. Furthermore, the liver produces thrombopoeitin, which is responsible for platelet production from megakaryocytes. Thrombocytopenia, as a result of decreased platelet production or increased platelet turnover and intravascular activation of hemostasis results in consumption of hemostatic factors, contributing to an alteration in the hemostatic system.

Portal hypertension, which is always present in end stage liver disease patients, plays an important role in coagulopathy by reducing the number of circulating platelets and in the development of collateral circulation. All the above mentioned factors contribute to an increased risk of hemorrhage during surgery. Usually, therapy for

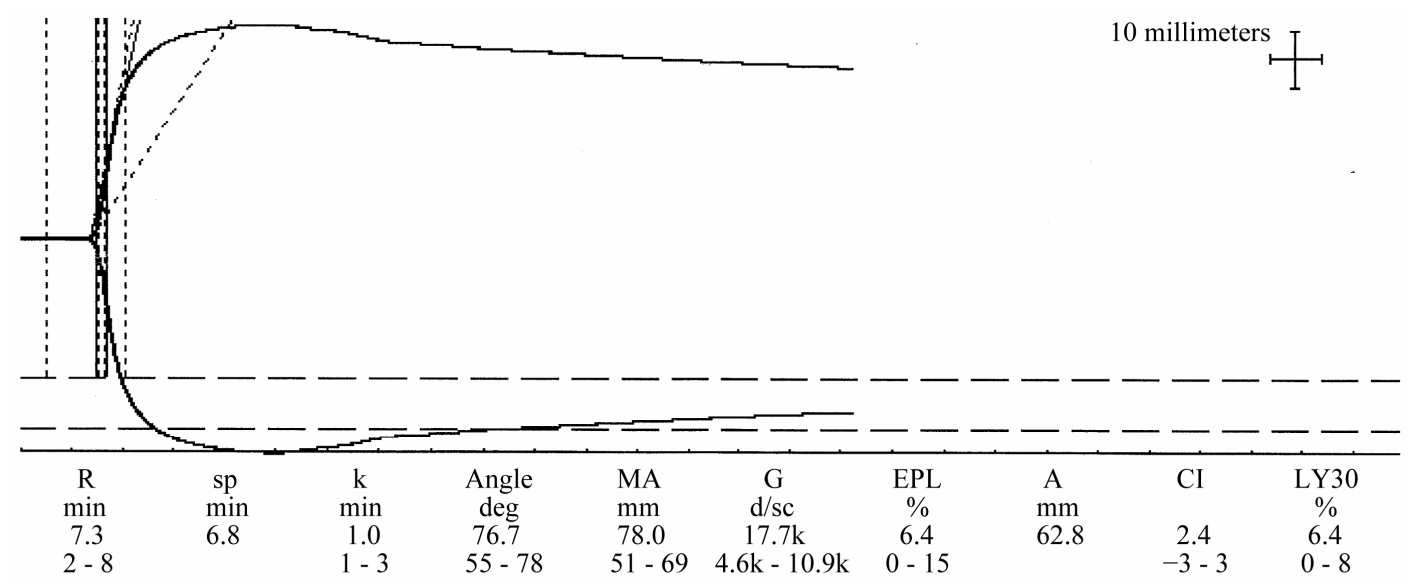

Figure 1. Pre-operative thrombelastogram. 
coagulation disorders in liver disease is needed only during bleeding episodes or before invasive procedures. When end stage liver disease occurs, liver transplant is the only treatment available which can restore normal hemostasis.

Preoperative standard monitoring of coagulation before liver transplantation consists of INR, PT, PTT, (International normalized ratio, Prothrombin time, Partial Thromboplastin time) platelets count and fibrinogen level.

Our patient required chronic dual anti platelet therapy to prevent thrombosis of two drug eluting coronary artery stents that were placed eight months prior to LDLT. Although platelet number remains within normal range both clopidogrel and aspirin produce platelet dysfunction. The antithrombotic effect of aspirin depends on irreversible inhibition of cyclooxygenase, which leads to decrease of thromboxane A2 formation and reduces platelet aggregation. Clopidogrel is a pro-drug. Its antiaggregating activity is caused by a short lasting metabolite generated in the liver by the cytochrome P 450-dependent pathway. This metabolite, 2-oxo-clopidogrel is further oxidized and then irreversibly binds to low affinity ADP platelet receptors. Many studies have shown that it is not universally effective and when effective there is significant pharmacogenetic variability in the population. $[7,8]$. The mechanisms leading to variability in clopidogrel responsiveness are multifactorial. High pre-treatment platelet activity, differences in individual absorption, as well as level of its active metabolite and possible drug interactions have shown to reduce clopidogrel responsiveness [9]. In 2009, a boxed warning was issued by the FDA on clopidogrel estimating that $2 \%-14 \%$ of the population are poor metabolizers of clopidogrel and are less likely to achieve a clinically therapeutic outcome [10]. Other clinical factors that may lead to reduction of clopidogrel effects include lack of drug prescription, poor compliance, and inappropriate dosing. With this in mind it is crucial to systematically evaluate platelet function in all patients on dual platelet therapy prior to major urgent or emergent surgery.

Thromboelastography (TEG) and platelet mapping are useful assays of platelet function that may be obtained prior to LDLT. Thromboelastography measures the speed and strength of clot formation including initial reaction time, speed of clot formation, clot strength and fibrinolysis. A new thromboelastographic essay Platelet Mapping measures the percentage of platelet inhibition and aggregation in the blood of the patients taking aspirin, arachidonic acid antagonist, or clopidogrel an ADP receptor antagonist. Platelet mapping assays specifically determine MA (maximum amplitude) reduction present with antiplatelet therapy. Two methods of measuring platelet function were used in order to confirm the accuracy of the tests. TEG monitoring has been noted to be unreliable and unable to differentiate clinically significant differences in platelet function in samples that have been treated with GP IIb/IIIa inhibitors in vitro [11]. Together, TEG and platelet mapping can be used to quantitate the degree of platelet function in any patient on dual anti platelet therapy allowing an informed clinical decision about the necessity of preoperative platelet transfusion for LDLT or other major surgery. In this case, TEG analysis revealed normal clot strength, normal fibrin formation, normal clot growth rate and clot stability. In order to determine the degree of platelet suppression from aspirin and clopidogrel, platelet mapping was done. Platelet mapping showed a normal rate of clot formation and clot strength for both drugs. Preoperative platelet transfusion was deemed unnecessary in this case.

Platelet transfusion during LDLT has been associated with increased morbidity and mortality. Pereboom et al. studied 449 patients undergoing orthotopic liver transplantation in the Netherlands. Patients who received platelet transfusions had lower one year graft survival than those who were not transfused (69\% vs 85\%). One year survival rate was $74 \%$ in the group receiving platelet transfusion and $92 \%$ in those who were not transfused. The increased rate of graft demise was felt to be due to the higher overall mortality in the transfusion group rather than a specific adverse effect of transfused platelets on the grafted liver. They also noted an eleven fold increase in the incidence of acute lung injury in patients who received platelet transfusion. Acute lung injury remained the main determinant of increased mortality [12,13].

One prior case study of a patient undergoing cadaveric liver transplant on dual anti platelet therapy for stroke has been reported in 2012. The patient was transfused with two units of platelets preoperatively and received another unit during the surgery as well as six units of packed red blood cells and six units of fresh frozen plasma. After massive transfusion the patient's course was complicated by myoglobinuria [14]. The authors do not report any results of perioperative TEG or platelet mapping study, and they do not comment on the indications to the blood, platelets or fresh frozen plasma transfusion.

Our case is the first report of living donor liver transplant for the patient with coronary artery stents on dual antiplatelet therapy with aspirin and clopidogrel. Our experience shows that LDLT can be performed safely in a patient on dual antiplatelet therapy. Careful preoperative evaluation of coagulation, including studies of platelet function allow informed decisions about perioperative blood and blood products transfusions. Avoidance of un- 
necessary transfusion will improve patient outcome.

\section{REFERENCES}

[1] Emond, J.C., Whitington, P.F., Thistlewaite, J.R., Alonso, E.M. and Broelsch, C.E. (1989) Reduced-size orthotopic liver transplantation: Use in the management of children with chronic liver disease. Hepatology, 10, 867-872. doi:10.1002/hep.1840100520

[2] Broelsch, C.E., Burdelski, M.D., Rogiers, X., Gundlach, M., Knoefel, W.T., Langwieler, T., Fischer, L. and Latta, A. (1994) Living donor for liver transplantation. Нераtology, 20, 49S-55S.

[3] Nadalin, S., Bockhorn, M., Malago, M., Valentin-Gamazo, C., Frilling, A. and Broelsch, C.E. (2006) Living donor liver transplantation. Hepato-Pancreato-Biliary Association, 1, 10-21.

[4] Lentine, K.L., Costa, S.P. and Weir, M.R. (2012) Cardiac disease evaluation and management among kidney and liver transplant candidates. A scientific statement from AHA/ACCF. Journal of American College of Cardiology, 60, 434-480. doi:10.1016/j.jacc.2012.05.008

[5] Levine, G.N., Bates, E.R., Blankenship, J.C., Bailey, S.R., Bittl, J.A., Cercek, B. and Chambers, C.E. (2011) ACCF/ AHA/SCAI Practice Guideline for Percutaneous Coronary Intervention Executive summary. Circulation, 124, 25742609.

[6] A report by American Society of Anesthesiologists Committee on Standards and Practice Parameters (2009) Practice alert for perioperative management of patients with coronary artery stents. Anesthesiology, 110, 22-23.

[7] Gurbel, P.A., Bliden, K.P., Hiatt, B.L. and O’Connor, C.M. (2003) Clopidogrel for coronary stenting: Response variability, drug resistance, and the effect of pretreatment platelet reactivity. Circulation, 107, 2908-2913. doi:10.1161/01.CIR.0000072771.11429.83
[8] Angiolillo, D.J., Fernandez-Ortiz, A., Alfonso, F., Bernardo, E.B., Macaya, C., Bass, T.A. and Costa, M.A. (2007) Variability in individual responsiveness to clopidogrel. Clinical implications, management, and future prospectives. Journal of Am. Collage of Cardiology, 49, 1505-1516. doi:10.1016/j.jacc.2006.11.044

[9] Taubert, D., Kastrati, A., Harlfinger, S., Gorchakova, O., Lazar, A., von Beckerath, N., Schmig, A. and Schomig, E. (2004) Pharmacokinetics of clopidogrel after administration of a high loading dose. Thrombosis and Haemostasis, 92, 311-316.

[10] FDA Drug Safety Communication (2010) Reduced effectiveness of plavix (clopidogrel) in patients who are poor metabolizers of the drug. http://www.fda.gov

[11] Bailey, L.A., Sistino, J.J. and Uber, W.E. (2005) Is platelet function as measured by Thrombelastograph monitoring in whole blood affected by platelet inhibitors? Journal of Extra Corporeal Technology, 37, 43-47.

[12] Khan, H., Belsher, J., Yilmaz, M., Afessa, B., Winters, J.L., Moore, S.B., Hubmayr, R.D. and Cajic, O. (2007) Fresh-frozen plasma and platelet transfusions are associated with development of acute lung injury in critically ill medical patients. Chest, 131, 1308-1314. doi:10.1378/chest.06-3048

[13] Pereboom, I.T., de Boer, M.T., Haagsma, E.B., Hendriks, G.D., Lisman, T. and Porte, R.J. (2009) Platelet transfusion during liver transplantation is associated with increased postoperative mortality due to acute lung injury. Anesthesia \& Analgesia, 108, 1083-1091. doi:10.1213/ane.0b013e3181948a59

[14] Spieker, H., Benckert, C., Quante, M., Thelen, A., Gaebelein, G., Kaisers, U. and Jonas, S. (2012) Successful liver transplantation with continued dual antiplatelet therapy. Annals of Transplantation, 17, 127-130. doi:10.12659/AOT.883231 